\title{
Tuning to Real-Life Language Statistics: Online Processing of Multiword Sequences in Native and Non-Native Speakers Across Language Registers
}

\author{
Elma Kerz ${ }^{\mathrm{a}}$, Daniel Wiechmann ${ }^{\mathrm{b}}$, Felicity F. Frinsel ${ }^{\mathrm{c}}$, Morten H. Christiansen ${ }^{\mathrm{c}}$

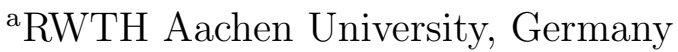 \\ ${ }^{\mathrm{b}}$ University of Amsterdam, Netherlands \\ ${ }^{\mathrm{c}}$ Cornell University, USA
}

\begin{abstract}
A large body of research over the past two decades has demonstrated that children and adults are equipped with statistical learning mechanisms that facilitate their language processing and boost their acquisition. However, this research has been conducted primarily using artificial languages that are highly simplified relative to real language input. Here, we aimed to determine to what extent adult native and non-native speakers show sensitivity to real-life language statistics obtained from large-scale analyses of authentic language use. Through a within-subject design, we conducted a series of behavioral experiments geared towards assessing the sensitivity to two types of distributional statistics (frequency and entropy) during online processing of multiword sequences across four registers of English (spoken, fiction, news and academic language). Our results show that both native and non-native speakers are able to 'tune to' multiple distributional statistics inherent in different types of real language input.
\end{abstract}

\section{Introduction}

One of the major advances in the language sciences across theoretical orientations has been in recognizing that natural languages are abundant in statistical and distributional regularities or patterns (Chater \& Christiansen, 2016; Gibson et al. 2019). Learning a language is considered to heavily involve figuring out regularities or extracting patterns inherent in the input; such statistical knowledge is seen to constitute an essential part of what it means to know a language (see, e.g., Ellis, 2019). This is supported by a growing body of evidence from the literature on statistical learning (SL), defined as a mechanism, largely implicit in nature, by which language users discover the structure of language based on its distributional properties - such as frequency, variability, distribution and co-occurrence probability (for reviews, see, e.g., Romberg \& Saffrans, 2010; Erickson \& Thiessen, 2015; Frost, Armstrong \& Christiansen, 2019; Rebuschat \& Monaghan, 2019).

Until recently, statistical learning has been studied using primarily artificial language learning or sequence learning paradigms that allow the isolation of specific input characteristics and 
distributional patterning. At the same time, calls have been made to integrate real world statistics in carefully controlled experimental designs (Pelucchi, Hay, and Saffran, 2009; Matthews and Bannard, 2010; Johnson and Tyler, 2010). This is mainly due to a growing awareness that, in comparison to artificial stimuli, the inputs we receive from natural languages rarely display statistical structure or patterns with the same clear consistency. Additionally, learning about the statistical regularities and distributional patterns inherent in the ambient language is a continuous process that does not end at some discrete point in time during ontogenetic development but instead takes place across the lifespan (e.g., Wells, Christiansen, Race, Acheson \& MacDonald, 2009; Saffran \& Kirkham, 2018; Frost, Armstrong \& Christiansen, 2019).

In contrast to early child language acquisition (prior to literacy), where children are mainly exposed to the statistics of spoken linguistic input (i.e., to child-directed speech), the role of written language becomes increasingly more important in later stages of learning (after the onset of formal education) which also sees increased demands on literacy (reading and writing). Indirect support for this assumption comes from a growing number of studies indicating that written language constitutes a key input type in the development of linguistic knowledge, as it provides a source of substantial change in the statistical make-up of an individual's language experience (e.g., Montag \& MacDonald, 2015; Seidenberg \& MacDonald, 2018). Language users are thus faced with the challenge of keeping track of the ever-changing statistics of these two main types of language input. This challenge is exacerbated by considerable variability in the distributional properties of linguistic patterns and structures at multiple levels inherent in spoken and written language, as demonstrated in numerous corpus-based studies on register ${ }^{1}$ variation (Roland, Dick Elman, 2007; Neumann, 2014; for a substantial contribution to this line of research, see, work by Biber and colleagues, e.g., Biber \& Conrad, 2019; Goulart et al., 2020).

In this paper, we report on a series of experiments designed to determine whether and to what extent native and non-native speakers develop sensitivity towards register-specific distributional statistics of language patterns by performing computational and statistical analyses of large samples of corpus data representing authentic language use in four registers of English: spoken language, written fiction, print news, and academic writing. We used the results from these analyses to carefully construct the stimulus material and derive predictions for response latencies in online processing of multiword sequences (MWS). MWS - also referred to as 'formulaic language' - is a cover term used for various types of recurrent continuous or discontinuous sequences of

\footnotetext{
${ }^{1}$ The term register is commonly used to refer to language variation in relation to communicative purposes and situational context.
} 
words (see, Wray, 2002; Christiansen \& Arnon, 2017 for overviews).

MWS have been the subject of increased attention in contemporary theories of language learning and processing. This interest stems from an extensive body of evidence demonstrating that both child and adult populations, including adult second-language learner populations, can develop the sensitivity to the statistics of MWS and rely on such statistics to facilitate language processing and acquisition (for overview, see for example, Shaoul \& Westbury, 2011; Ellis, 2011; Arnon \& Christiansen, 2017). This line of research has demonstrated how the statistics obtained from corpora of authentic language use can be successfully employed to predict language behavior across different types of experimental designs (i.e., accuracy rates, reaction times, and eye movement patterns). However, this research has been confined to assessing sensitivity to distributional statistics of one type of language input, namely spoken language (for studies in child language acquisition, see, Bannard \& Matthews, 2008; Matthews \& Bannard, 2010; for studies on adult both first and second language - processing see, Arnon, McCauley \& Christiansen, 2017; Arnon \& Snider, 2010; Hernandez et al., 2016; Kerz \& Wiechmann, 2017; Yi et al., 2017). In this paper, we emphasize the importance of taking into account variability of natural languages in developing the stimulus material used in behavioral experiments, as a desirable methodological addition to SL research in the domain of language comprehension (see Section 'The present study').

A significant body of research has demonstrated that frequency is the most robust statistic among different kinds of distributional information to drive the acquisition and processing of language in both child and adult populations (see, Ambridge, Kidd, Rowland \& Theakston, 2015; Conklin \& Schmitt, 2012 for reviews). Frequency effects have been consistently observed across multiple components of language, including that of sound/grapheme/word combinations, morphemes and syntactic structures (see Diessel, 2007; Ellis, 2002 for reviews; see also Divjak \& Gries, 2012 and Gries \& Divjak, 2012 ). Three mechanisms have been proposed to underpin frequency effects: (1) increased frequency causes the strengthening of linguistic representations, (2) increased frequency causes the strengthening of expectations and (3) increased frequency leads to the automatization of chunks. The frequency with which patterns of language occur is thus an important driving force behind chunking and, all else being equal, each exposure to a given sequence of sounds, graphemes or words will affect its subsequent processing. But why is there a need for chunking? To ameliorate the effects of the 'real-time' constraints on language processing imposed by the limitations of the human sensory system and memory in combination with the continual deluge of language input (cf. Christiansen \& Chater, 2016a,b, for the 'Now-or-Never 
bottleneck'), through constant exposure to (both auditory and visual) language input, humans learn to rapidly and efficiently recode incoming information into larger sequences. Moreover, the degree to which as MWS form a meaningful unit further contributes to and helps reinforce its ease of processing (Jolsvai, McCauley \& Christiansen, 2020). Statistical learning and chunking also play an important role in the anticipatory language processing that humans rely on to integrate the greatest possible amount of available information as fast as possible.

In the area of MWS, numerous studies have examined processing advantages of more frequent strings of words following two different approaches (see Arnon \& Snider, 2010, for more details on the approaches). In the threshold-approach, the stimulus material is typically derived from language corpora based on predefined frequency criteria to demonstrate advantages only for sequences above a particular frequency threshold (see, e.g., Tremblay et al., 2012). In the second approach, a continuous effect of frequency employed to demonstrate incremental advantages for all sequences spanning the full frequency spectrum after controlling for substring frequency (Arnon \& Snider, 2010; Arnon, McCauley \& Christiansen, 2017; Hernandez et al., 2016; Kerz \& Wiechmann, 2017). Apart from token frequency (i.e., how often a particular language pattern occurs in the input), type frequency (i.e., how many distinct items can appear in a given slot in a pattern) has also figured in the literature on the acquisition of language structure through statistical learning (see, Tomasello, 2003, and the role of type frequency in child language acquisition; for its role in second language acquisition, see, e.g., Ellis, 2019).

The general assumption in this literature is that a schematic pattern is likely to be extracted from the input in cases where some invariant structure is combined with a wide range of other material. Gomez (2002), for example, demonstrated that toddlers' ability to detect a nonadjacent dependency between two sounds is predicted by the degree of variability of the intervening material in the artificial language to which they were exposed. Similar mechanisms have been proposed for the acquisition of productive patterns in child language through a process of schematization (Tomasello, 2003; for earlier work on this, see Braine's pivot grammar, 1963). A schematic productive pattern (It's time for $\mathrm{X}$ ) is likely to emerge when children are repeatedly exposed to a specific language form (e.g., It's time for) in their ambient language along with varied use of another form (e.g. noun phrases referring to what whatever is time for: It's time for lunch, It's time for soup, It's for drums, It's time for bed) across similar situational contexts. Type frequency is thus used to quantify the variability of a given slot in a pattern and correspondingly the likelihood that a schematic pattern will be extracted from the ambient language. 
However, as was pointed out by Matthews and Bannard (2010), one problem with type frequency is that it does not take into account the frequency distribution of the possible fillers of a given slot. They illustrate this with the following example: Consider the scenario where a child hears the sequence Throw your bottle 118 times and Throw the ball and Throw teddy only once each, versus the scenario where each of the three MWS are heard 40 times. While in each scenario the type frequency would have been three, the likelihood of the child extracting a potential 'Throw $\mathrm{X}$ ' pattern might differ. In the first ('unequal') scenario, the child would expect to hear your bottle to follow throw making it less likely that a child would extract a productive pattern, whereas in the second scenario, the child is uncertain as to which of three possible fillers are likely to appear in the slot and thus might be more likely to form a productive slot. Matthews \& Bannard (2010) argued that the intuitive difference between the two scenarios can be adequately captured with an information-theoretic measure of the entropy (Shannon and Weaver, 1949) of the slot, an index of the uncertainty regarding which of all the possible words that could fill a slot is most likely to occur. In their study, testing the effects of entropy on 2- to 3-year-old childrens' processing of MWS, they found that children were more likely to correctly repeat unfamiliar sequences with high slot entropy. To our knowledge, this is the only study to test such entropy effects on real time processing of MWS. Our second goal in this paper is therefore to assess whether and to what extent the entropy effects can also be observed in adult populations.

As mentioned above, there are now numerous studies showing that both native and non-native speakers can develop sensitivity to the distributional properties of MWS. These studies suggest that adult language learners retain the statistical learning ability in second language learning and processing and may share a general statistical learning mechanism with native speakers when processing MWS (e.g., Hernandez et al., 2016; Yi, Lu \& Ma, 2017; Kerz \& Wiechmann, 2017). One question that still remains to be addressed is whether and to what extent native speakers and their non-native peers exhibit similar patterns of sensitivity towards multiple statistics of MWS inherent in different registers. In addressing this research question, we provide empirical evidence and further clarifications relevant for the debate on whether the mechanisms underlying first language (L1) and second language (L2) learning are fundamentally different - calling for separate mechanisms (e.g., Bley-Vroman, 2009) - or whether similar processes underlie both types of learning (MacWhinney, 2012). 


\section{The present study}

In a nutshell, the aim of the current paper is threefold. Our first aim is to examine to what extent adult (native and non-native) speakers are sensitive to real-world distributional patterns of language characterized by variability contingent upon language registers. On the methodological level, we aim to demonstrate that such statistics obtained through computational and statistical analyses of large samples of authentic language use can be utilized in experimental studies. Our second aim is to take into account not only the effects of token frequencies ('more simple' distributional statistics) but also consider the effects of 'more complex' distributional statistics (entropy) that capture the productivity of language patterns. Our third aim is to seek evidence for the generality of distributional mechanisms underlying learning and processing beyond one's L1 extending it to an L2.

In this paper, we focus on the role of statistical knowledge in real-time processing of MWS - i.e. four-word combinations - measured by distributional frequencies and entropy values. Whereas the former operationalizes the probability of encountering a given sequence, and is hence an estimate of how likely a sequence may occur in the language input, the latter is an expression of the slot variability and the probability of the retrieval of a schematic pattern. Building on previous empirical findings reported in the literature on processing of such sequences (Arnon and Snider, 2010; Matthews and Bannard, 2010; Hernandez et al., 2017), we predict shorter response latencies for higher frequency MWS, compared to lower frequency control items, and for items with higher entropy values in the final slot position, compared to items with lower entropy values.

To address our three aims, we performed analyses of large samples of corpus data representing four registers and used the results from these analyses to make predictions about language users' performance in a series of MWS decision tasks. In these tasks, participants were visually presented with four-word sequences drawn from corpora representing different language registers (spoken, news, fiction, and academic language) and asked to judge - as quickly and accurately as possible

- if a given four-word sequence that appeared on the screen was a possible sequence in English or not. Our study employed a MWS frequency (continuous; within-subjects) x entropy (highmid-low; within-subjects) x register (spoken, news, fiction, academic; within-subjects) x language status (L1/L2; between-subjects) design. 


\section{Method}

\subsection{Participants}

Sixty English native speakers (L1 group; mean age $=19.57$ years, $\mathrm{SD}=1.32$ years) and sixty German native speakers, with English as their second language (L2 group; mean age $=23.56$ years, $\mathrm{SD}=4.52$ years) participated in the study. All participants were college students recruited from the Cornell University (L1 group) and RWTH Aachen University (L2 group). All non-natives were classified as having a Common European Framework of Reference for Language (CEFR) English proficiency level of at least B2 (upper intermediate) or C1 (lower advanced) based on their institutional status, as they all graduated with the German 'Abitur' (higher education entrance qualification). We also estimated their proficiency level through the LexTALE test (Lemhöfer \& Broersma, 2012), an English vocabulary size test that is often used to estimate the CEFR proficiency level. The average score of 73.21 corresponds to the B2 skill level, labeled 'vantage or upper intermediate' proficiency level in the CEFR. Participants in the L2 group were also asked to fill the Language Experience and Proficiency Questionnaire (LEAPQ), a validated questionnaire tool for collecting self-reported proficiency and experience data from bilingual and multilingual speakers ages 14 to 80 (for a recent overview, see Kaushanskaya, Blumenfeld, \& Marian, 2019). The data gathered from the LEAP-Q and the LexTALE test are reported in Table 1, showing means, standard deviations and observed ranges of the L2 group.

\subsection{Materials}

Pairs of four-word sequences were created, differing only in their final word and in overall MWS frequency (high vs. low) but matched for substring frequency (e.g. to justify the cost vs. to justify the effort; e.g., is beyond the scope vs. is beyond the boundaries). A total of 240 experimental items were constructed (60 for each of the four registers), using the Corpus of Contemporary American English (COCA; Davies, 2008), a 560 million words corpus with approximately equalsized subcomponents representing the statistics of MWS from our four target registers: (1) spoken (118 million words), (2) fiction (113 million words), (3) newspaper (114 million words) and (4) academic journals (112 million words). In a first step, all COCA text files were preprocessed using the sentence splitting (ssplit) and tokenization (PTNTokenizer) components from the Stanford CoreNLP toolkit V.3.2.9 (Manning et al., 2014). In a second step, frequencies for all n-grams of orders 1 to 4 were extracted using Java scripts. N-grams with a frequency of one were discarded. 
Table 1: Self-report information on English acquisition, exposure, and proficiency of the non-native group of participants

\begin{tabular}{lccc}
\hline & mean & sd & obs. range \\
\hline English acquisition (years) & & & \\
Age start acquisition & 8.46 & 2.23 & $6-22$ \\
Age became fluent & 14.63 & 3.9 & $8-23$ \\
Current exposure to English & & & \\
Friends (0-10) & 4.63 & 3.1 & $0-10$ \\
Family (0-10) & 1.36 & 2.6 & $0-10$ \\
Reading (0-10) & 7.64 & 2.25 & $1-10$ \\
Class instruction (0-10) & 5.48 & 3.43 & $0-10$ \\
Self instruction (0-10) & 4.86 & 2.81 & $0-10$ \\
Watch (0-10) & 7.64 & 2.72 & $0-10$ \\
Listening music (0-10) & 7.39 & 2.84 & $0-10$ \\
Social Media (0-10) & 7.39 & 2.68 & $0-10$ \\
Immersion (month) & & & \\
English speak. country & 2.96 & 3.46 & $0-11$ \\
LexTALE & 73.21 & 10.64 & $53.75-95$ \\
Self-rated proficiency & & & \\
Speaking (0-10) & 7.25 & 1.69 & $1-10$ \\
Listening (0-10) & 8.49 & 1.38 & $5-10$ \\
Reading (0-10) & 7.86 & 1.58 & $1-10$ \\
\hline
\end{tabular}

These two steps were performed on the RWTH Aachen University high-performance computing cluster. To ensure that the position at which entropy was measured was filled by a lexical word, four-grams ending with a function word were filtered out in a third step. The Shannon entropy $\mathrm{H}$ was computed for the final word slot of all remaining four-grams, calculated with the formula shown in (1). $\mathrm{X}$ is the final slot of the MWS, the unit of $\mathrm{x}$ represents a word that appears in that slot, and $\mathrm{p}(\mathrm{x})$ is the probability of seeing each $\mathrm{x}$ in that position. All conditional word probabilities needed to compute entropy scores were estimated using second-order Markov models (cf. Willems, Frank, Nijhof, Hagoort, \& van den Bosch, 2015).

$$
H(X)=-\sum_{x} p(x) \log p(x)
$$

Next, all four-word sequences that shared the first three words were selected across the four registers investigated here. Within each set of such four-word sequences, a frequency difference score (FDS) was computed for a given sequence in relation to the most frequent sequence in that set. After sequences were ordered according to their FDS a moving window technique was used to explore how FDS scores related to entropy. A window with a size that corresponded to a predefined FDS was moved over the entire candidate item pool to bin all four-gram into groups with similar FDS (see Figure 1 for a visualization). Inspection of these data indicated that 
four-grams with small differences in FDS tended to exhibit low entropy scores. Based on these observations, we restricted our candidate pool to four-grams that had entropy scores between 0 and 3 and a difference in log normalized frequency between 6.5 and 30. Finally, from each register, a total of 60 experimental item pairs were randomly sampled: 20 pairs from each of three entropy ranges ('low': $\mathrm{H}(\mathrm{X})$ between 0 and 1, 'mid': $\mathrm{H}(\mathrm{X})$ between 1 and 2, 'high': $\mathrm{H}(\mathrm{X})$ greater than 2). Consequently, by applying these filters, the log frequencies of our items ranged between 0.69 and 6.85 (spoken 0.69 - 6.07, fiction: 0.69 - 6.85; news 0.69-5.97, academic $0.69-5.87$ ). The stimulus material will be made available at the Open Science Framework (https://osf.io/).

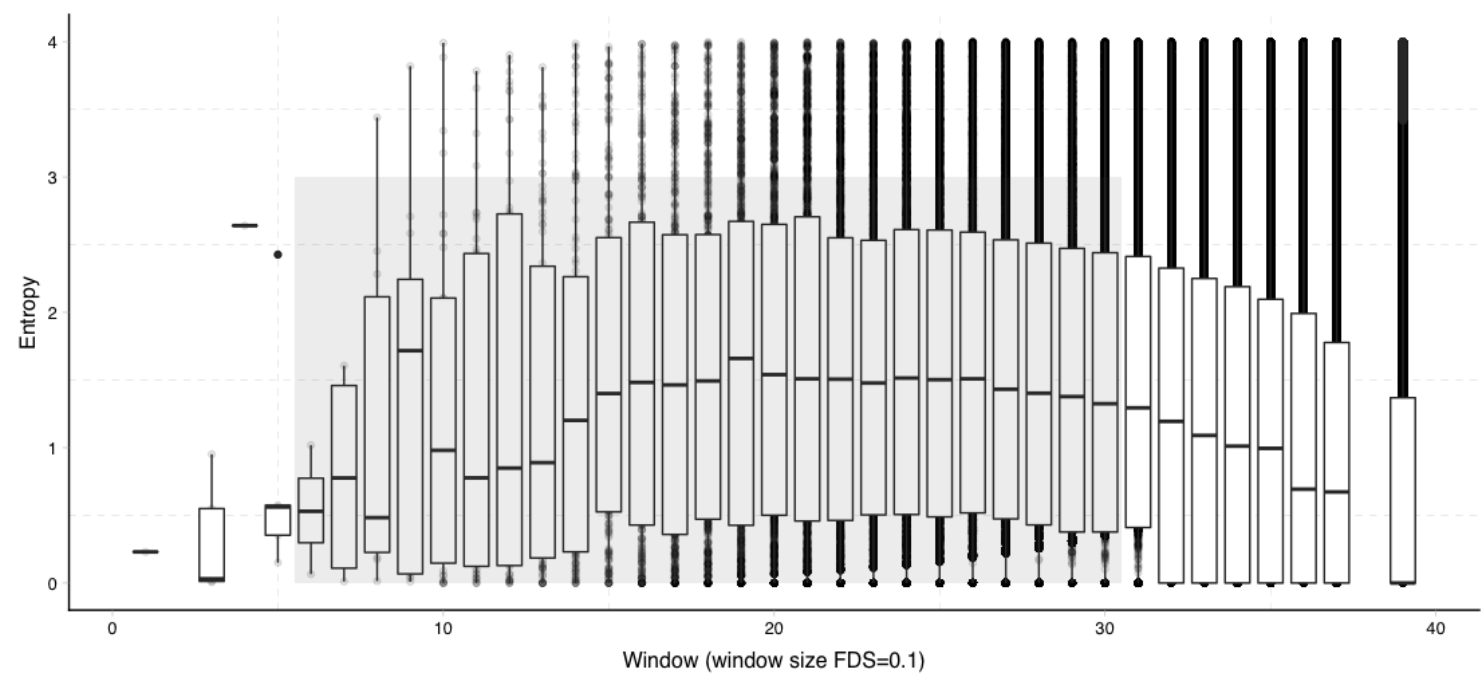

Figure 1: Distribution of entropy scores across the 'frequency difference score' (FDS) range for the academic sub-component of Corpus of Contemporary American English (Davies, 2008). The shaded area represents the range from which experimental items were sampled.

\subsection{Procedure}

The L1 group was tested on all four language registers within a single session in the Cognitive Neuroscience Laboratory in the Department of Psychology at Cornell University. The L2 group was tested on two different days, with two language registers tested per day (Day 1: academic and fiction; Day 2: news and spoken) in the lab facility of the Department of English Linguistics at RWTH Aachen University. Each language register experiment was divided into two blocks of about 7 minutes each with a short break between blocks. The 120 four-word MWS from a given register were distributed across two lists, each containing one of the two variants of a given pair. As a consequence, participants would never see both variants of a pair. In addition to the experimental items, each list also contained 60 ungrammatical items with a scrambled word order. 
The order of presentation of the blocks was counterbalanced between participants.

In this task, participants were asked to judge if a four-word sequence that appeared on the screen was a possible sequence in English or not. This is essentially a phrasal version of the classic lexical decision task (for prior use of the phrasal decision task, see e.g., Arnon Snider, 2010; Arnon et al., 2017; Jolsvai et al., 2020). Each trial began with the presentation of a fixation point for $500 \mathrm{~ms}$. The MWS appeared at once in the middle of the screen and participants were instructed to respond as quickly and accurately as possible using the keyboard. The MWS stayed visible on the screen until participants responded or until $3000 \mathrm{~ms}$ had passed. The task was implemented and run in PsychoPy v3.0 (Peirce et al., 2019), an open-source software package for creating rich, dynamic experiments in psychology, neuroscience and linguistics.

\section{Results}

Participants in both groups were highly accurate in their responses to MWS and fillers - independent of register (overall accuracy rates were $>90 \%$, see Table 2). Only items that were correctly identified as a possible sequence in English and had a response time greater than $200 \mathrm{~ms}$ but shorter than two SD from the mean were included. After applying these criteria, only a small percentage of data in each group was lost $(\mathrm{L} 1=5.9 \%$; $2=7.2 \%)$.

Table 2: Accuracy rates (\%) per experimental item and fillers in each group of participants across registers in the MWS task.

\begin{tabular}{lccc}
\hline Register & Item type & Native & Non-native \\
\hline Academic & Exp. item & 0.98 & 0.96 \\
Academic & Filler & 0.81 & 0.89 \\
\hline Fiction & Exp. item & 0.96 & 0.97 \\
Fiction & Filler & 0.87 & 0.88 \\
\hline Spoken & Exp. item & 0.94 & 0.93 \\
Spoken & Filler & 0.86 & 0.88 \\
\hline News & Exp. item & 0.94 & 0.95 \\
News & Filler & 0.83 & 0.90 \\
\hline TOTAL & & 0.9 & 0.92
\end{tabular}

Linear mixed-effects models were used for all analyses and were implemented with the lme4 (v1.1-21, Bates et al., 2015) and lmerTest (v3.1-0, Kuz-netsova et al, 2017) packages in the R environment (version 3.6.1; R Core Team, 2017). All models were fitted by restricted maximum likelihood (REML). P-values were derived using Satterthwaite approximations. Log response latencies were used as the predicted variable to reduce the skewness in the distribution. As participants' response latencies were expected to be affected by the substring frequencies of the MWS, their ef- 
fects were factored out by including the frequency of the final word, bigram and trigram of a given MWS as control variables into the models. To avoid introducing multicollinearity into our models, frequency counts of the substring frequencies were transformed to orthogonal dimensions using principal components analysis (henceforth, the terms 'PC1', 'PC2', 'PC3' refer to the first second and third principal component, respectively). The models further contained MWS length (in characters, continuous) and block order (2 levels, first vs. second block in a testing session) as additional covariates. As these five control variables were not manipulated, their effects are reported in the results tables (regardless of their significance) but are not discussed. In all analyses, collinearity between the fixed effects was checked by calculating generalized variance-inflation factors (GVIF, Fox and Monette, 1992). All reported models had low collinearity, $\left(G V I F^{(1 /(2 * d f)} \leq 2.3\right.$, where $d f$ is the number of coefficients in the subset).

To assess whether adult native and non-native speakers show sensitivity to MWS frequency and entropy across the four target language registers, we first fitted separate models to the log response times (RT) of each register (spoken, news, fiction, and academic) for each group (L1 and L2 speakers). All models included random intercepts for participants and items, as well as by-subject random slopes for log MWS frequency and slot entropy, respectively 2 The results of the models are summarized in Tables 3 and 4 .

In the L1 group, the effect of MWS frequency was significant in three out of the four target language registers. More specifically, after controlling for substring frequency, MWS length, and block order, significantly shorter RT latencies were observed for higher frequency MWS in the spoken register $(\beta=-0.0420, S E=0.0103, t=-4.047, p<0.001)$, in the news register $(\beta=$ $-0.0364, S E=0.0112, t=-3.268, p=0.0014)$, and in the fiction register $(\beta=-0.0228, S E=$ $0.01132, t=-2.013, p=0.046)$. In the academic register, RT latencies were numerically shorter for higher frequency MWS, however this effect was not statistically significant ( $\beta=-0.0164, S E=$ $0.0107, t=-1.538, p=0.123)$. In the L2 group, significant effects of MWS frequency were found the spoken register $(\beta=-0.0264, S E=0.0084, t=-3.104, p=0.002)$, the news register $(\beta=-0.0228, S E=0.0093, t=-2.434, p=0.016)$ and the academic register $(\beta=-0.015, S E=$ $0.0073, t=0.942, p=0.556)$. However, no significant effect was found in the fiction register $(\beta=-0.0053, S E=0.0088, t=-0.608, p=0.556)$. These findings replicated the MWS frequency effect found in child, adult L1 (Bannard \& Matthews, 2008; Arnon \& Snider, 2010), and adult

\footnotetext{
${ }^{2}$ The results of model comparisons using likelihood ratio tests revealed that the inclusion of by-subject random slopes did in most cases not significantly improve model fit relative to a random-intercepts model. However, given recommendation to keep the random effects structure close to maximal (Barr, Levy, Scheepers, \& Tily, 2013), we report the results here of models that included by-subject random slopes for both key predictors.
} 
L2 populations (Hernandez et al., 2016; Kerz \& Wiechmann, 2017) in spoken (conversational) English. Importantly, the results extend previous research by demonstrating that sensitivity to MWS frequency is not restricted to spoken language but is also evident in written registers of the English language for both native and non-native speakers.

Having considered how the effects of token frequencies impact online processing of MWS, we now turn to the question of whether RT latencies are affected by the entropy of the word sequences at the final word position. After controlling for the effects of frequency of the final word, bigram and trigram (via the inclusion of PC1, PC2 and PC3) as well as the length of the MWS and block order, mixed effects modeling revealed only a significant effect of slot entropy (at a significance level $\alpha=0.05)$ in the news register $(\beta=0.0365, S E=0.0176, t=2.074, p=0.04)$ in the L1 group. The effect in the spoken register $(\beta=0.0299, S E=0.0175, t=1.713, p=0.089)$, however, was marginally significant. In the L2 group, the entropy effect in the news register was found to be marginally significant $(\beta=0.0228, S E=0.0136, t=1.675, p=0.097)$. A-gainst our predictions, RT latencies were found to increase with increasing entropy. However, visual inspection of the relationship between entropy and response latency suggests that the direction of the effect changes across the entropy range (see Figure 2(b)): In both the L1 and the L2 group, response latencies appear to increase with increasing entropy in the lower-entropy range $(0<H<2)$, but decrease with increasing entropy in the higher-entropy range $(H>2)$. To further explore this observation, additional models were fitted in which entropy was coded as a three-level categorical variable distinguishing the three entropy bins used to define the sample space of the MWS (see section 3.2 Materials). For the relevant register (news writing), the analysis revealed a significant increase in response latency associated with a change in entropy from lower-entropy $(0<H \leq 1)$ to medium-entropy $(1 \leq H<2)(\beta=0.096, S E=0.0351, t=2.672, p=0.0078)$. However, the change in response latency associated with a change from lower-entropy to higher-entropy $(H \geq 2)$ was not statistically significant $(\beta=0.0179, S E=0.0361, t=0.496, p=0.62)$, indicating that participants were just as fast in responding to MWS with low and high entropy. The nonlinear effects of entropy on response latencies in the news registers were also supported by the results of models that included polynomial terms of entropy. Specifically, model comparisons with added polynomial terms of entropy of increasing degree (up to cubic order) revealed that in these registers the entropy effect was best captured by a quadratic model (news: $\operatorname{Chi}(1)=13.58, p=0.0002$; fiction: $\operatorname{Chi}(1)=6.81, p=0.009)$, giving rise to the inverted U-shapes shown in Figure 2.

Finally, to assess whether different performance patterns between the two groups yielded 


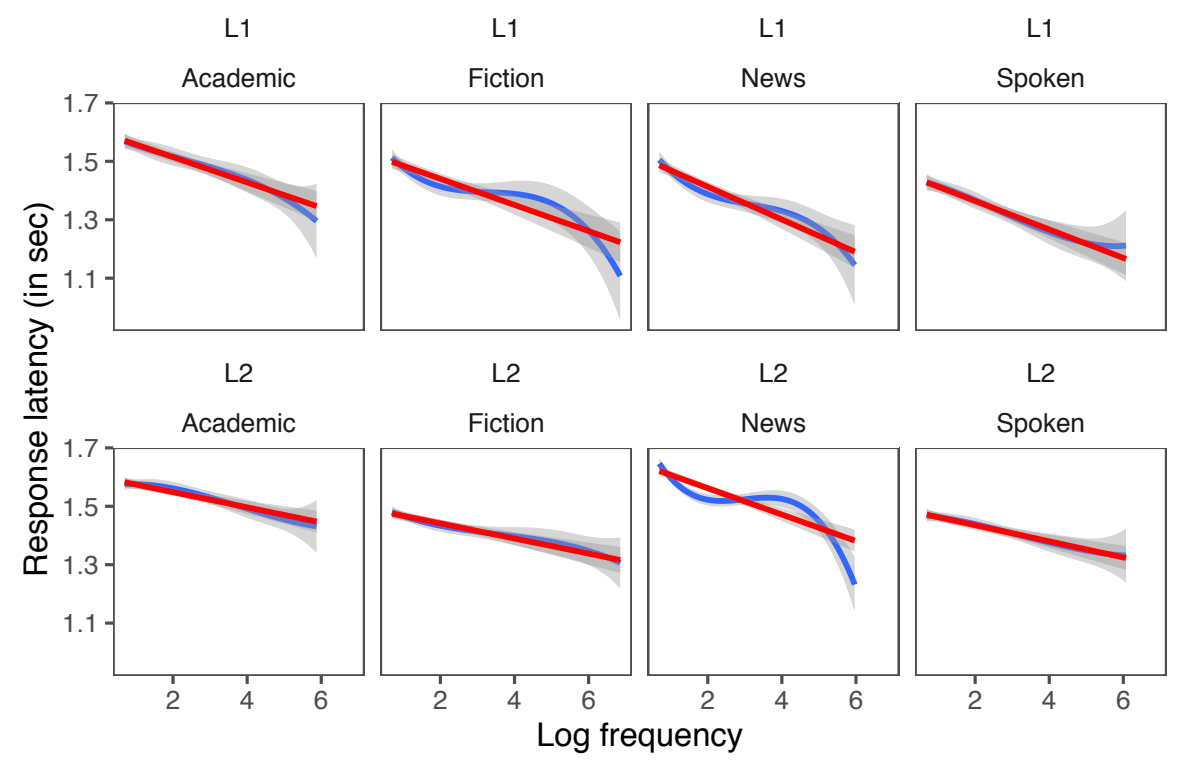

(a) Response latency (raw) as a function of log MWS frequency across groups and registers.

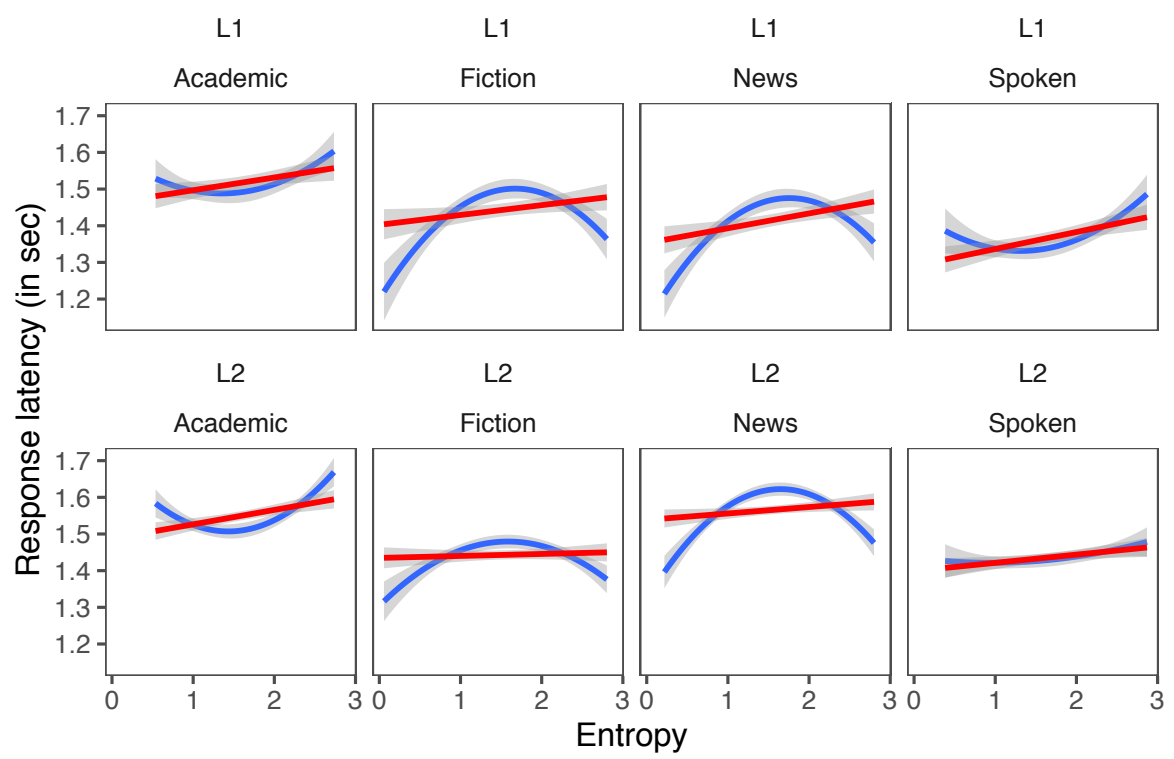

(b) Response latency (raw) as a function of entropy cross groups and registers.

Figure 2: Response latency (raw) as a function of log MWS frequency (left) and entropy (right) across groups and registers. Red lines represent a linear model; blues lines show the results of a quadratic model. The shaded areas represents the $95 \%$ confidence level intervals for predictions from the respect models. 
Table 3: Results of linear mixed-effects models calculated to estimate the effects of $(\log )$ MWS frequency on RT latencies (in $\log$ seconds); Linear mixed model fit by REML; Formula: $\log (\mathrm{RT}) \sim \log$ MWSfreq $+\mathrm{PC} 1+\mathrm{PC} 2+\mathrm{PC} 3+$ LengthPhrase + BlockOrder $+(1+$ $\log$ MWSfreq|Participant $)+(1 \mid$ Phrase $)$

\begin{tabular}{lcccccccc}
\hline \hline & \multicolumn{7}{c}{ Dependent variable: (log) RT latencies } \\
\cline { 2 - 9 } & Spoken & News & Fiction & Academic & Spoken & News & Fiction & Academic \\
\hline \multirow{2}{*}{ Intercept } & 0.167 & 0.110 & 0.180 & 0.047 & $0.322^{* * *}$ & $0.394^{* * *}$ & $0.515^{* * *}$ & $0.223^{* * *}$ \\
& $(0.136)$ & $(0.118)$ & $(0.143)$ & $(0.115)$ & $(0.083)$ & $(0.059)$ & $(0.077)$ & $(0.049)$ \\
MWS Freq & $-0.042^{* * *}$ & $-0.037^{* * *}$ & $-0.023^{* *}$ & -0.017 & $-0.026^{* * *}$ & $-0.023^{* *}$ & -0.005 & $-0.015^{* *}$ \\
& $(0.010)$ & $(0.011)$ & $(0.011)$ & $(0.011)$ & $(0.008)$ & $(0.009)$ & $(0.009)$ & $(0.007)$ \\
\hline PC1 & $-0.033^{* * *}$ & 0.006 & -0.017 & -0.015 & $0.013^{*}$ & -0.012 & $0.016^{*}$ & -0.008 \\
& $(0.008)$ & $(0.010)$ & $(0.011)$ & $(0.010)$ & $(0.007)$ & $(0.008)$ & $(0.009)$ & $(0.007)$ \\
PC2 & $-0.034^{* *}$ & 0.010 & 0.012 & -0.013 & -0.006 & -0.018 & $0.022^{* *}$ & $-0.021^{* * *}$ \\
& $(0.015)$ & $(0.014)$ & $(0.013)$ & $(0.011)$ & $(0.012)$ & $(0.011)$ & $(0.010)$ & $(0.007)$ \\
PC3 & -0.014 & -0.003 & 0.035 & 0.022 & 0.005 & -0.008 & $0.056^{* * *}$ & 0.008 \\
& $(0.019)$ & $(0.019)$ & $(0.023)$ & $(0.019)$ & $(0.015)$ & $(0.015)$ & $(0.018)$ & $(0.013)$ \\
Length MWS & 0.004 & $0.006^{* *}$ & 0.002 & $0.010^{* * *}$ & 0.005 & $0.007^{* * *}$ & -0.0002 & $0.009^{* * *}$ \\
& $(0.004)$ & $(0.003)$ & $(0.005)$ & $(0.002)$ & $(0.004)$ & $(0.002)$ & $(0.004)$ & $(0.002)$ \\
Block Order & 0.042 & 0.062 & 0.065 & 0.057 & -0.025 & $-0.061^{* * *}$ & $-0.097^{* * *}$ & 0.010 \\
& $(0.060)$ & $(0.059)$ & $(0.067)$ & $(0.059)$ & $(0.018)$ & $(0.018)$ & $(0.021)$ & $(0.014)$ \\
\hline \hline Note: & \multicolumn{7}{c}{$(0.010)$} \\
\hline
\end{tabular}

Table 4: Results of linear mixed-effects models calculated to estimate the effects of entropy on response time latencies (in log seconds); Linear mixed model fit by REML; Formula: $\log (\mathrm{RT}) \sim$ Entropy + PC1 + PC2 + PC3 + LengthPhrase + BlockOrder + (1 + Entropy|Participant $)+(1 \mid$ Phrase)

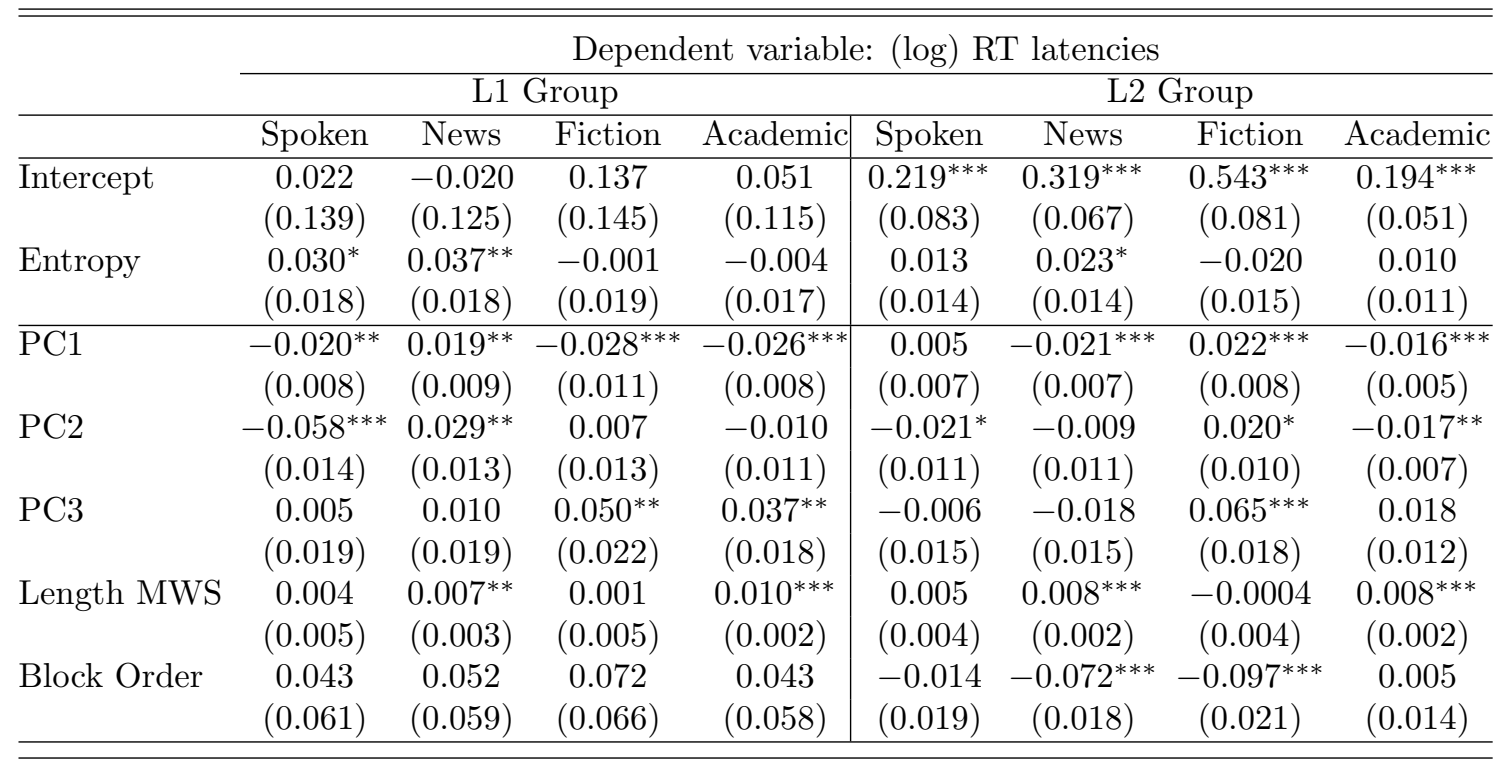

Note:

${ }^{*} \mathrm{p}<0.1 ;{ }^{* *} \mathrm{p}<0.05 ;{ }^{* * *} \mathrm{p}<0.01$

higher-level interactions with the factor group, an omnibus model to the response time data across L1 and L2 speakers was fitted. This model included main effects of both key predictors 
(MWS frequency and entropy), the two grouping variables (register and group), and the five control variables (the three substring frequency control PC1-3, length of the MWS and block order), as well as all two- and three-way interactions between the two grouping variables and each key predictor. The categorical grouping variables were dummy coded with the academic register as the reference level for register and L1 as the reference level for group. The continuous key predictors, MWS frequency and entropy, were mean-centered prior to being entered into the models to mitigate the effects of multicollinearity introduced by the inclusion of the interaction (e.g. inflated standard errors and indeterminacy among the parameter estimates). The results of the omnibus model are shown in Table 5. The analysis revealed several significant interactions. First, there was a marginally significant effect of group $(\beta=0.0643, S E,=0.0343, t=1.8750, p=0.0632)$ qualified by a significant interaction between group and register indicating that the difference in response latencies between the L1 and the L2 groups was more pronounced in the spoken register $(\beta=0.0298, S E, 0.0076, t=0.897, p<0.001)$ and news register $(\beta=0.0298, S E=0.0076$, $t=0.897, p<0.001)$, but not in the fiction register $(\beta=0.0092, S E=0.0078, t=1.1770$, $p=0.2392)$, relative to the academic register. Second, a significant interaction between MWS frequency and group indicated that the frequency effect was overall stronger in native speakers $(\beta=0.0139, S E=0.0083, t=-2.94, p=0.0034)$. The three-way interaction between MWS Frequency, group and register was not significant. Third, the omnibus model revealed a marginally significant interaction between group and entropy $(\beta=0.0298, S E=0.0076, t=0.897, p<0.001)$ indicating that L2 speakers' response latencies were impacted by the entropy to a lesser extent than was the case in the L1 groups. This effect was qualified by significant three-way interaction between entropy, group and register, indicating that group differences in the entropy effect were most pronounced in the fiction register (in the L2 group, the effect of entropy was near zero).

\section{Discussion}

Our study was geared towards determining to what extent adult native and non-native speakers show sensitivity to real-world distributional statistics of the English language in a series of behavioral experiments using a within-subject design. The statistics were characterized by variability found in different (spoken and written) language registers. These statistics were obtained through the application of techniques of Natural Language Processing and statistical analyses of samples of natural language use from a large-scale representative corpus of English. The results of these 
Table 5: Results of the full (omnibus) mixed effects regression model $(\log (\mathrm{RT}) \sim$ cent $\log$ MWS freq + cent entropy + Register + Language + Language $\times$ Register + Register $\times$ cent log MWS freq + Language $\times$ cent $\log$ MWS freq + Register $\times$ cent entropy + Language $\times$ cent entropy + Language $\times$ Register $\times$ cent log MWS freq + Language $\times$ Register $\times$ cent entropy + PC1 + PC2 + PC3 + cent length + BlockOrder $+(1+$ cent $\log$ MWS freq + cent entropy $\mid$ participant $)+(1 \mid$ Phrase))

\begin{tabular}{|c|c|c|c|c|c|}
\hline$\overline{\overline{\text { Random effects }}}$ & Name & Variance & SD & Correlation & \\
\hline Item & (Intercept) & 0.0100 & 0.0998 & & \\
\hline \multirow[t]{3}{*}{ Subject } & (Intercept) & 0.0341 & 0.1846 & & \\
\hline & MWS Freq & 0.0001 & 0.0079 & -0.2900 & \\
\hline & Entropy & 0.0002 & 0.0148 & -0.15 & 0.0900 \\
\hline Residual & & 0.0951 & 0.3084 & & \\
\hline Fixed effects & $\beta$ & SE & $\mathrm{t}$ & $\operatorname{Pr}(>|t|)$ & \\
\hline$\overline{\text { (Intercept) }}$ & 0.3725 & 0.0275 & 13.5640 & $0 \quad 0.0000$ & $* * *$ \\
\hline \multicolumn{6}{|l|}{ Main effects of group and register } \\
\hline Group L2 & 0.0643 & 0.0343 & 1.8750 & 0.0632 & . \\
\hline Register Fiction & -0.0285 & 0.0152 & -1.8740 & 0.0615 & . \\
\hline Register News & -0.0575 & 0.0145 & -3.9770 & 0.0001 & $* * *$ \\
\hline Register Spoken & -0.0915 & 0.0148 & -6.2040 & 0.0000 & $* * *$ \\
\hline \multicolumn{6}{|l|}{ Interactions between group and register } \\
\hline Register Fiction $\times$ Group L2 & 0.0092 & 0.0078 & 1.1770 & 0.2392 & \\
\hline Register News $\times$ Group L2 & 0.0842 & 0.0076 & 11.1390 & 0.0000 & $* * *$ \\
\hline Register Spoken $\times$ Group L2 & 0.0298 & 0.0076 & 3.8970 & 0.0001 & $* * *$ \\
\hline \multicolumn{6}{|l|}{ Main effects of key predictors } \\
\hline MWS Freq & -0.0244 & 0.0083 & -2.9400 & 0.0034 & $* *$ \\
\hline Entropy & -0.0019 & 0.0160 & -0.1220 & 0.9028 & \\
\hline \multicolumn{6}{|l|}{ Interactions with group } \\
\hline MWS Freq $\times$ Group L2 & 0.0139 & 0.0043 & 3.1880 & 0.0015 & $* *$ \\
\hline Entropy $\times$ Group L2 & 0.0154 & 0.0091 & 1.7000 & 0.0895 & e \\
\hline \multicolumn{6}{|l|}{ Interactions with register } \\
\hline MWS Freq $\times$ Register Fiction & 0.0018 & 0.0109 & 0.1640 & 0.8702 & \\
\hline MWS Freq $\times$ Register News & -0.0093 & 0.0113 & -0.8270 & 0.4085 & \\
\hline MWS Freq $\times$ Register Spoken & -0.0009 & 0.0109 & -0.0810 & 0.9357 & \\
\hline Entropy $\times$ Register Fiction & 0.0028 & 0.0217 & 0.1310 & 0.8958 & \\
\hline Entropy $\times$ Register News & 0.0193 & 0.0220 & 0.8770 & 0.3811 & \\
\hline Entropy $\times$ Register Spoken & 0.0098 & 0.0220 & 0.4470 & 0.6549 & \\
\hline \multicolumn{6}{|l|}{ Three-way interactions with language and register } \\
\hline MWS Freq $\times$ Register Fiction $\times$ Group L2 & -0.0026 & 0.0060 & -0.4380 & 0.6612 & \\
\hline MWS Freq $\times$ Register News $\times$ Group L2 & -0.0088 & 0.0060 & -1.4600 & 0.1442 & \\
\hline MWS Freq $\times$ Register Spoken $\times$ Group L2 & -0.0050 & 0.0061 & -0.8180 & 0.4135 & \\
\hline Entropy $\times$ Register Fiction $\times$ Group L2 & -0.0327 & 0.0121 & -2.7030 & 0.0069 & $* *$ \\
\hline Entropy $\times$ Register News $\times$ Group L2 & -0.0264 & 0.0118 & -2.2450 & 0.0248 & * \\
\hline Entropy $\times$ Register Spoken $\times$ Group L2 & -0.0270 & 0.0121 & -2.2430 & 0.0249 & $*$ \\
\hline \multicolumn{6}{|l|}{ Control variables } \\
\hline PC1 & 0.0029 & 0.0043 & 0.6770 & 0.4986 & \\
\hline $\mathrm{PC} 2$ & 0.0011 & 0.0057 & 0.2010 & 0.8405 & \\
\hline PC3 & 0.0087 & 0.0086 & 1.0130 & 0.3115 & \\
\hline Length (in words) & 0.0072 & 0.0014 & 5.0060 & 0.0000 & $* * *$ \\
\hline BlockOrder & -0.0321 & 0.0054 & -5.9450 & 0.0000 & $* * *$ \\
\hline
\end{tabular}


analyses formed the basis for a careful construction of the stimulus material used to investigate online processing of multiword sequences (MWS) across four language registers: (1) spoken, (2) fiction, (3) news and (4) academic language. The aim of the present study was threefold: First, we aimed to assess sensitivity to token frequencies of longer word combinations (four-word sequences) across the four language registers in the two target populations. Second, we examined whether this sensitivity extends to more complex distributional statistics measured by the (slot) entropy of the final word position. And third, we determined whether and to what extent native and non-native speakers exhibit similar patterns of sensitivity towards the multiple statistics of MWS.

Our findings indicated that both native and non-native speakers show sensitivity to token frequencies of MWS, as evidenced by shorter response latencies to more frequent sequences. Importantly, we observed such effects in three out of four registers investigated in this study: In all but the academic register for the L1 group and in all but the fiction register for the L2 group. These findings corroborate and extend those of previous studies demonstrating facilitatory effects of sequence frequency in online processing of longer (four- and five-word) sequences with sub-string frequencies being controlled for in both child and adult native speaker populations (Bannard and Matthews, 2008; Arnon \& Snider, 2010, Tremblay et al. 2011; Arnon, McCauley \& Christiansen, 2017), and adult second-language learner populations (Hernandez et al. 2016; Kerz \& Wiechmann, 2017).

Importantly, however, to the best of our knowledge, the present study is the first to demonstrate that these effects are not limited to spoken language but can also be observed in written language. This finding is of particular interest as recent psycholinguistic and statistical learning literature emphasize that written language is an essential source of input, introducing substantial change in the statistics of an individual's language experience that differ considerably from those of spoken language. This literature indicates that the amount of exposure to written language and its statistical properties is a strong predictor of the development of adult-like language behavior (Montag \& MacDonald, 2015; Montag, Jones \& Smith, 2015, 2018) and - among other cognitive factors - of individual differences in native language attainment (Dabrowska, 2018) and second language processing (Kerz \& Wiechmann, 2020). Thus, a comprehensive assessment of human sensitivity to the statistics in natural languages needs to take the statistical patterns of written language into account, when constructing stimulus material. The finding that both L1 and L2 speakers exhibit differential sensitivity to sequence frequency contingent upon language register emphasizes the importance of integrating research on statistical learning and language (Misyak \& 
Christiansen, 2012; Frost, Armstrong \& Christiansen, 2019; Bogaerts, Frost \& Christiansen, 2020) with research on register and its relationship to language use and variation (Biber \& Conrad, 2019; Grey \& Egbert, 2019; Neumann, 2013). Given that we observed heterogeneity in effect sizes in both groups (standardized beta coefficients for L1 group: $\beta_{\text {spoken }}=-0.132, \beta_{\text {news }}=-0.115, \beta_{\text {fiction }}=$ $-0.072, \beta_{\text {academic }}=-0.057 ;$ standardized beta coefficients for L2 group: $\beta_{\text {spoken }}=-0.097, \beta_{\text {news }}=$ $\left.-0.095, \beta_{\text {fiction }}=-0.023, \beta_{\text {academic }}=-0.064\right)$, the question remains as to what extent these effects are determined by the amount of experience an individual has had with specific registers of spoken and written language, and their interactions with cognitive and affective individual differences factors (see, Kidd, Donnelly \& Christiansen, 2017). For instance, there is initial evidence that the online processing of MWS is impacted by cognitive factors, such as statistical learning ability (Kerz and Wiechmann, 2019) as well as working memory capacity and executive function (Kerz \& Wiechmann, 2020). Processing differences across L1 and L2 speakers may also be affected by differential sensitivity to the semantics of the MWS (see Jolsvai, McCauley \& Christiansen, in press, for L1 evidence).

Our findings with respect to the second aim are not entirely in line with prior expectations: We observed a significant effect of entropy on response latencies only in one of the four language registers - that of newspaper articles - and only in the native speaker group. Moreover, the entropy effect was found to be nonlinear, such that response latencies first became longer when entropy increased from the low range $(H<1)$ to the mid range $(1<H<2)$ but then became shorter when entropy increased further to the high range $(H>2)$. This pattern was found in both groups under investigation. Our findings on entropy effects are to a certain extent consistent with the ones reported in Matthews \& Bannard (2010). As in our study, they used stimuli that were matched on all sub-sequence frequencies except the final one, two, or three words. For each of the initial three words they calculated the slot entropy for the final word's slot. As word sequences with a high slot entropy value exhibit increased uncertainty for what word appears in that slot, their assumption was that greater slot entropy increases the likelihood that a child forms a basic ('low level') generalization (a schema), which in turn can facilitate language processing. However, they found such facilitatory effects only for unfamiliar sequences but not for familiar ones. The authors suggested that this may be due to peculiarities in their very small stimulus set (9 triplets of four-word sequences from three entropy ranges). However, our stimulus set (see Section 3.2) was carefully constructed using a moving-window approach simultaneously capturing frequency and entropy ranges and included considerably more items than the Matthews and Bannard's study, 60 
pairs of four-word sequences from three entropy ranges (120 items per register), suggesting that the conditional nature of the effect is not attributable to the size of the stimulus set. Rather it appears that human language processing is facilitated at certain entropy ranges: either in the low or high range. Further research is needed to elucidate the role of entropy on online processing of MWS geared towards systematically studying the relative frequency of possible slot fillers and the skewness of such distributions.

Finally, regarding the third aim, our data contributes to a long-standing debate that lies at the heart of research into multilingualism surrounding the question of whether L1 and L2 learning and processing are subserved by different mechanisms or are qualitatively similar. Our results imply that the similarities between L1 and L2 processing are more striking than the differences, which is in line with the results reported by Hernandez et al. (2016), Kerz \& Wiechmann (2017) and Onnis \& Yun (2020) This calls for unified theoretical models of L1 and L2 learning and processing that highlight a common reliance on mechanisms of statistical learning and cumulative knowledge of statistical regularities acquired through the exposure to a target language (Christiansen \& Chater, 2016a; MacWhinney, 2012; Ellis, 2019). Our findings speak to the growing recognition and importance of fostering a bilateral integration of statistical learning research with cognitive science (Frost et al., 2019; see, Bogaerts, Frost \& Christiansen, (2020) for a recent special issue in JML; see also contributions to the invited panel at CogSci 2020 on statistical learning and development).

Even though extracting statistics from large-scale corpus resources representing authentic language use is computationally intense, the inclusion of statistics obtained from such corpora in constructing the stimulus material to be used in language processing tasks is of importance for one simple reason: It more adequately represents the complexities of the real-world distributional statistics inherent in natural languages - thereby better reflecting the actual types of input humans are exposed to - and going beyond the simple uniform distributions traditionally used in artificial language learning paradigms (for recent exceptions, see, Lavi-Rotbain \& Arnon, 2020; Li et al., 2020; Snell \& Theeuwes, 2020). Our approach also has the potential to link statistical learning research more tightly with that of a related field of research on language adaptation (for an overview, see, Chang et al, 2012). Assessing sensitivity to real-world statistics of both spoken and written language and its registers across different age groups may provide unique insights into experientially-driven lifelong adaptive processes shown to occur across multiple levels of language representations (ranging from phonemes, syllables - in case of auditory input - or from graphemes 
- in case of written input - to words, multiword sequences and higher-level syntactic structures). Here the focus was on multiword sequences but our approach is easily extendable to other types of building blocks of language. For instance, building on Roland et al. (2007), Kerz and Wiechmann (2019) demonstrated how register-contingent variability in distributional statistics of complex sentences with relative clauses obtained from a large-scale representative corpus of English can be linked to production choices.

\section{Conclusion}

Our study made notable theoretical and methodological contributions to advancing our understanding of the links between real-world distributional statistics and its impact on human language processing. Future research is needed to scale up the approach taken in this study so as to generate large amounts of data from a broader and more diverse population through crowdsourcing (see, Suchow, Griffiths and Hartshorne, 2020) and to obtain reliable approximations of the amount and types of language experience different demographics are likely to be exposed to at different stages across their lifespan. The latter requires an integrative, multi-method approach combining estimates of an individual's exposure to spoken and written language, computational simulations of learning environments and statistics of additional emerging language registers (Biber and Egbert, 2018) that portray the sources of language input in a more nuanced way.

\section{References}

Ambridge, B., Kidd, E., Rowland, C. F., \& Theakston, A. L. (2015). The ubiquity of frequency effects in first language acquisition. Journal of child language, 42(2), 239-273.

Arnon, I., \& Christiansen, M. H. (2017). The role of multiword building blocks in explaining 11-12 differences. Topics in Cognitive Science, 9(3), 621-636.

Arnon, I., McCauley, S. M., \& Christiansen, M. H. (2017). Digging up the building blocks of language: Age-of-acquisition effects for multiword phrases. Journal of Memory and Language, 92, 265-280.

Arnon, I., \& Snider, N. (2010). More than words: Frequency effects for multi-word phrases. Journal of Memory and Language, 62(1), 67-82. 
Bannard, C., \& Matthews, D. (2008). Stored word sequences in language learning: The effect of familiarity on children's repetition of four-word combinations. Psychological Science, 19(3), $241-248$.

Barr, D. J., Levy, R., Scheepers, C., \& Tily, H. J. (2013). Random effects structure for confirmatory hypothesis testing: Keep it maximal. Journal of Memory and Language, 68(3), 255-278.

Biber, D., \& Conrad, S. (2019). Register, genre, and style. Cambridge University Press.

Biber, D., \& Egbert, J. (2018). Register variation online. Cambridge University Press.

Bley-Vroman, R. (2009). The evolving context of the fundamental difference hypothesis. Studies in Second Language Acquisition, 175-198.

Braine, M. D. (1963). The ontogeny of english phrase structure: The first phase. Language, 1-13.

Chang, F., Janciauskas, M., \& Fitz, H. (2012). Language adaptation and learning: Getting explicit about implicit learning. Language and Linguistics Compass, 6(5), 259-278.

Christiansen, M. H., \& Arnon, I. (2017). More than words: The role of multiword sequences in language learning and use. Topics in cognitive science, 9(3), 542-551.

Christiansen, M. H., \& Chater, N. (2016a). Creating language: Integrating evolution, acquisition, and processing. Cambridge, MA: MIT Press.

Christiansen, M. H., \& Chater, N. (2016b). The Now-or-Never bottleneck: A fundamental constraint on language. Behavioral and Brain Sciences, 39, e62.

Conklin, K., \& Schmitt, N. (2012). The processing of formulaic language. Annual Review of Applied Linguistics, 32.

Davies, M. (2009). The 385+ million word Corpus of Contemporary American English (1990-2008+): Design, architecture, and linguistic insights. International Journal of Corpus Linguistics, $14(2), 159-190$.

Divjak, D., \& Gries, S. T. (2012). Frequency effects in language representation (Vol. 244). Walter de Gruyter.

Ellis, N. C. (2011). Implicit and explicit sla and their interface. Implicit and explicit language learning: Conditions, processes, and knowledge in SLA and bilingualism, 35, 47.

Ellis, N. C. (2019). Essentials of a theory of language cognition. The Modern Language Journal, 103, 39-60.

Erickson, L. C., \& Thiessen, E. D. (2015). Statistical learning of language: Theory, validity, and predictions of a statistical learning account of language acquisition. Developmental Review, $37,66-108$. 
Frost, R., Armstrong, B. C., \& Christiansen, M. H. (2019). Statistical learning research: A critical review and possible new directions. Psychological Bulletin, 145(12), 1128.

Gibson, E., Futrell, R., Piantadosi, S. P., Dautriche, I., Mahowald, K., Bergen, L., \& Levy, R. (2019). How efficiency shapes human language. Trends in cognitive sciences, 23(5), 389-407.

Gomez, R. L. (2002). Variability and detection of invariant structure. Psychological Science, $13(5), 431-436$.

Goulart, L., Gray, B., Staples, S., Black, A., Shelton, A., Biber, D., ... Wizner, S. (2020). Linguistic perspectives on register. Annual Review of Linguistics, 6, 435-455.

Gries, S. T., \& Divjak, D. (2012). Frequency effects in language learning and processing (Vol. 244). Walter de Gruyter.

Hernández, M., Costa, A., \& Arnon, I. (2016). More than words: multiword frequency effects in non-native speakers. Language, Cognition and Neuroscience, 31(6), 785-800.

Jolsvai, H., McCauley, S. M., \& Christiansen, M. H. (2020). Meaningfulness beats frequency in multiword chunk processing. Cognitive Science, 44 (10), e12885.

Kaushanskaya, M., Blumenfeld, H. K., \& Marian, V. (2019). The language experience and proficiency questionnaire (leap-q): Ten years later. Bilingualism: Language and Cognition, $1-6$.

Kerz, E., \& Wiechmann, D. (2017). Individual differences in L2 processing of multi-word phrases: Effects of working memory and personality. In R. Mitkov (Ed.), Computational and corpusbased phraseology. EUROPHRAS 2017 (pp. 306-321).

Lavi-Rotbain, O., \& Arnon, I. (2020). The learnability consequences of zipfian distributions: Word segmentation is facilitated in more predictable distributions.

Lemhöfer, K., \& Broersma, M. (2012). Introducing lextale: A quick and valid lexical test for advanced learners of english. Behavior Research Methods, 44(2), 325-343.

Li, R., Schatz, T., Matusevych, Y., Goldwater, S., \& Feldman, N. (2020). Input matters in the modeling of early phonetic learning in 463. In Proceedings of the 42nd annual meeting of the cognitive science society (Vol. 464).

MacWhinney, B. (2012). The logic of the unified model. In S. M. Gass \& A. Mackey (Eds.), The Routledge handbook of second language acquisition (pp. 211-227). Routledge London \& New York.

Manning, C., Surdeanu, M., Bauer, J., Finkel, J., Bethard, S., \& McClosky, D. (2014). The Stanford CoreNLP natural language processing toolkit. In Proceedings of 52nd annual meeting 
of the Association for Computational Linguistics: system demonstrations (pp. 55-60).

Matthews, D., \& Bannard, C. (2010). Children's production of unfamiliar word sequences is predicted by positional variability and latent classes in a large sample of child-directed speech. Cognitive Science, 34(3), 465-488.

Monaghan, P., Schoetensack, C., \& Rebuschat, P. (2019). A single paradigm for implicit and statistical learning. Topics in cognitive science, 11(3), 536-554.

Montag, J. L., \& MacDonald, M. C. (2015). Text exposure predicts spoken production of complex sentences in 8-and 12-year-old children and adults. Journal of Experimental Psychology: General, $144(2), 447-468$.

Neumann, S. (2013). Contrastive register variation: A quantitative approach to the comparison of english and german (Vol. 251). Walter de Gruyter.

Peirce, J., Gray, J. R., Simpson, S., MacAskill, M., Höchenberger, R., Sogo, H., ... Lindeløv, J. K. (2019). Psychopy2: Experiments in behavior made easy. Behavior research methods, $51(1), 195-203$.

Pelucchi, B., Hay, J. F., \& Saffran, J. R. (2009). Statistical learning in a natural language by 8-month-old infants. Child development, 80(3), 674-685.

Roland, D., Dick, F., \& Elman, J. L. (2007). Frequency of basic english grammatical structures: A corpus analysis. Journal of Memory and Language, 57(3), 348-379.

Romberg, A. R., \& Saffran, J. R. (2010). Statistical learning and language acquisition. Wiley Interdisciplinary Reviews: Cognitive Science, 1(6), 906-914.

Saffran, J. R., \& Kirkham, N. Z. (2018). Infant statistical learning. Annual Review of Psychology, $69,181-203$.

Seidenberg, M. S., \& MacDonald, M. C. (2018). The impact of language experience on language and reading: A statistical learning approach. Topics in Language Disorders, 38(1), 66-83.

Shannon, C. E., \& Weaver, W. (1949). The mathematical theory of communication. University of Illinois Press.

Shaoul, C., \& Westbury, C. (2011). Formulaic sequences: Do they exist and do they matter? The Mental Lexicon, 6(1), 171-196.

Snell, J., \& Theeuwes, J. (2020). A story about statistical learning in a story: Regularities impact eye movements during book reading. Journal of Memory and Language, 113, 104127.

Suchow, J., Griffiths, T., \& Hartshorne, J. (2020). Cogsci 2020 W6: Workshop on scaling cognitive science. 
Tomasello, M. (2003). Constructing a language. Harvard university press.

Tremblay, A., Derwing, B., Libben, G., \& Westbury, C. (2011). Processing advantages of lexical bundles: Evidence from self-paced reading and sentence recall tasks. Language learning, $61(2), 569-613$.

Wells, J. B., Christiansen, M. H., Race, D. S., Acheson, D. J., \& MacDonald, M. C. (2009). Experience and sentence processing: Statistical learning and relative clause comprehension. Cognitive Psychology, 58(2), 250-271.

Willems, R. M., Frank, S. L., Nijhof, A. D., Hagoort, P., \& Van den Bosch, A. (2015). Prediction during natural language comprehension. Cerebral Cortex, 26(6), 2506-2516.

Wray, A. (2013). Formulaic language. Language Teaching, 46(3), 316-334.

Yi, W., Lu, S., \& Ma, G. (2017). Frequency, contingency and online processing of multiword sequences: An eye-tracking study. Second Language Research, 33(4), 519-549. 